\title{
Evaluation eines ambulanten strukturierten Schulungsprogramms für Patienten mit COPD - eine Pilotstudie
}

Evaluation of a Structured Education Programme for Patients with COPD Under

Outpatient Conditions - a Pilot Study

\section{Zusammenfassung}

Für die chronische obstruktive Bronchitis mit und ohne Lungenemphysem (COPD) ist im Gegensatz zum Asthma bronchiale der Stellenwert der Patientenschulung im Management der Erkrankung nicht hinreichend geklärt. Daher wurde ein ambulantes strukturiertes Schulungs- und Behandlungsprogramm für Patienten mit chronisch obstruktiver Bronchitis entwickelt und in einer prospektiven, nichtkontrollierten Pilotstudie an 21 Patienten mit leicht- bis mittelgradiger COPD über 2 Jahre evaluiert. Wesentliche Inhalte des Programmes sind die Selbstkontrolle der Erkrankung mit Hilfe von Peak-Flow-Messung, Symptombeobachtung und Tagebuchführung, Kenntnisse über Wirkungen und Nebenwirkungen der Medikamente, korrekte Inhalationstechnik und Selbsthilfemaßnahmen bei einer Verschlechterung der Erkrankung. Die Häufigkeit von Episoden mit Atemnot nahm im Vergleich zu dem Jahr vor der Schulung (1,6 \pm 0,5 Episoden pro Patient) im ersten Jahr nach der Schulung $(1,0 \pm 0,5$; $\mathrm{p}<0,05)$ deutlich ab. Keine Veränderung ergab sich für die Gesamtzahl der schweren Exazerbationen, allerdings konnten mehr Exazerbationen durch Selbsthilfemaßnahmen der Patienten beherrscht werden. Weitere Effekte waren eine Zunahme des Wissens der Patienten über die Wirkungen und Nebenwirkungen der Medikamente: Vor der Schulung waren 9,1\% der Antworten in einem Multiple-Choice-Test korrekt, nach dem ersten Jahr 68,7\% ( $\mathrm{p}<0,01)$ und nach dem zweiten Jahr 70,5\% ( $<<0,01)$. Zudem konnte die Selbstkontrolle der Erkrankung durch Symptombeobachtung, Peak-Flow-Messung und Patiententagebuch verbessert werden. Die Anzahl der Krankenhaustage pro Jahr aufgrund der Diagnose COPD nahm signifikant von 10,1 $\pm 0,5$ Tage/Jahr auf 2,1 + 0,2 Tagen/Jahr $(\mathrm{p}<0,01)$ im ersten Jahr nach der Schulung ab und lag auch im zweiten Jahr nach der Schulung

\section{Abstract}

In contrast to other chronic diseases, such as bronchial asthma, the role of patient education in the management of COPD still remains unclear. Therefore, we evaluated the efficacy of a structured education programme for COPD-patients under outpatient conditions in a prospective pilot study including 21 patients with mild to moderate COPD over a period of two years. The main teaching items of the programme are: self-control of the disease with regular peak flow measurements, the observation of typical symptoms and the patients diary, effects and side effects of the medication, correct inhalation technique and the patients' ability to change the medication with respect to the actual degree of airflow limitation. The frequency of episodes of dyspnoea per day was significantly reduced after the first year. Before the training course the patients had an average of $1.6 \pm 0.5$ episodes of dyspnoea per day, after the first year $1.0 \pm 0.5$ $(p<0.05)$. With respect to the year before the intervention, the total number of the exacerbations did not change significantly in the two years after the participation in the programme, but the number of self-managed exacerbations increased. Further effects were an improvement of the knowledge of the patients about the effects and side effects of the medication: before the education the number of correct answers in a multiple choice test was $9.1 \%$, one year later $68.7 \%(\mathrm{p}<0.01)$ and after the second year $70.5 \%$ ( $p<0.01)$. The self-control of the disease could be improved by regular observation of symptoms, peak flow measurements and patients' diary. The number of hospital days due to the diagnosis COPD decreased from $10.1 \pm 0.5$ days per patient and year to $2.1 \pm 0.2$ days $(\mathrm{p}<0.01$ ) in the first year after the education and remained constant with $2.4 \pm 0.6$ days $(\mathrm{p}<0.01)$ even in the second year after the course. The efficacy of the education

\section{Institutsangaben \\ Medizinische Klinik I, Klinikum Fürth, Fürth \\ Korrespondenzadresse Dr. med. York Dhein · Medizinische Klinik I · Klinikum Fürth · Jakob-Henle-Str. 1 · 90766 Fürth}


konstant bei $2,4 \pm 0,6$ Tagen/Jahr $(p<0,01)$. Die Effektivität der Patientenschulung war auch noch zwei Jahre nach der Schulung nachweisbar, allerdings zeigte sich insbesondere bei der Selbstkontrolle der Erkrankung eine Abnahme der Schulungseffekte. Die Ergebnisse der Pilotstudie sprechen dafür, dass mittels einer strukturierten Patientenschulung das Management der leichtbis mittelgradigen chronisch obstruktiven Bronchitis verbessert werden kann. Kontrollierte Studien an größeren Patientenzahlen sind erforderlich, um den Stellenwert der Patientenschulung in der Behandlung der COPD zu bestimmen. programme is still evident two years after the training. In comparison to the results one year after the programme we found a loss of efficacy in some aspects, particularly in the self-control of the disease. In conclusion, structured education programmes under outpatient conditions could improve the self management abilities in patients with mild to moderate COPD. Randomized controlled trials with a larger sample size are needed to confirm the results of this pilot study.

\section{Einleitung}

Die chronisch obstruktive Bronchitis ist mit einer geschätzten Prävalenz von etwa 8\% [13] und jährlichen Krankheitskosten von ca. 5,5 Mrd. Euro in Deutschland [13] eine Erkrankung mit erheblicher sozialmedizinischer Bedeutung. Im Alter von mehr als 70 Jahren leidet jeder zweite Raucher und jeder fünfte NichtRaucher an dieser Erkrankung [20]. Ungeachtet der Fortschritte in Diagnose und Therapie $[27,33]$ ist die Mortalität unverändert hoch: In den USA ist die chronische Bronchitis mit 3,6\% aller Todesfälle die fünfthäufigste Todesursache, allerdings schwanken die Zahlen in verschiedenen Ländern erheblich. Deutschland nimmt einen mittleren Rang ein, man rechnet hier mit einer Letalität von 230 Männern und 60 Frauen pro 100000 Einwohnern [20,24]. Im Jahr 2020 wird Schätzungen zufolge die chronisch obstruktive Bronchitis weltweit die dritthäufigste Todesursache sein [13].

Für viele chronische Erkrankungen wie Diabetes mellitus, Hypertonie und Asthma bronchiale sind Patientenschulungen längst akzeptierter Bestandteil der Therapie [16]. Für Asthmatiker konnte die Effektivität ambulanter und stationärer Schulungsprogramme mehrfach belegt werden [15-19,22,23,29], während speziell für Patienten mit COPD zur Zeit keine evaluierten strukturierten ambulanten Schulungsprogramme im deutschsprachigen Raum zur Verfügung stehen [28].

Ziel dieser Pilotstudie war die Evaluation eines ambulanten und strukturierten Schulungsprogrammes für Patienten mit COPD im Rahmen einer Pilotstudie über einen Zeitraum von zwei Jahren.

\section{Material und Methoden}

\section{Patienten und Studienaufbau}

Insgesamt wurden 21 konsekutive Patienten (11 Frauen, 10 Männer) in die Studie eingeschlossen, für die Nachuntersuchungen nach einem Jahr standen alle Patienten zur Verfügung, für die Verlaufskontrolle nach zwei Jahren nur noch 19 Patienten (Ausfallrate: 9,5\%). Die Patienten wurden im Rahmen eines stationären Krankenhausaufenthaltes für die Studie rekrutiert. Die anthropometrischen Daten sowie die Kenngrößen der Lungenfunktion der Patienten sind in Tab. 1 enthalten. Zum Zeitpunkt der Schulung waren 13 Patienten aktive Raucher und 5 ehemalige Raucher, die Anzahl der „pack years“ betrug insgesamt 29,5 $\pm 4,3$.
Tab. 1 Anthropometrische Daten und lungenfunktionsanalytische Kenngrößen der 21 COPD-Patienten (Angaben in Mittelwert \pm Standardfehler SEM)

\begin{tabular}{|lll}
\hline & Mittelwert \pm SEM & Minimum - Maximum \\
\hline Alter (Jahre) & $65 \pm 2$ & $56-75$ \\
\hline Größe $(\mathrm{cm})$ & $166 \pm 2$ & $155-185$ \\
\hline Gewicht $(\mathrm{kg})$ & $77 \pm 3$ & $54-103$ \\
\hline $\mathrm{FEV}_{1}(\mathrm{l})$ & $1,6 \pm 0,1$ & $0,9-3,3$ \\
\hline $\mathrm{FEV} \mathrm{V}_{1} / \mathrm{VC}(\%)$ & $62 \pm 5$ & $51-79$ \\
\hline $\mathrm{R}_{\mathrm{AW}}(\mathrm{kPa} / \mathrm{l} / \mathrm{s})$ & $0,63 \pm 0,2$ & $0,3-0,8$ \\
\hline $\mathrm{PO}_{2}(\mathrm{~mm} \mathrm{Hg})$ & $74 \pm 5$ & $68-85$ \\
\hline $\mathrm{pCO}_{2}(\mathrm{~mm} \mathrm{Hg})$ & $38 \pm 6$ & $33-46$ \\
\hline Erkrankungsdauer (Jahre) & $7,0 \pm 0,5$ & $2,0-12,0$ \\
\hline aktive Raucher (n) & 13 & \\
\hline Ex-Raucher & 5 & \\
\hline pack years & $29,5 \pm 4,3$ & $9,0-55,0$ \\
\hline
\end{tabular}

Folgende Einschlusskriterien wurden aufgestellt: Neben der Diagnose „chronisch obstruktive Bronchitis“ müsste in der Lungenfunktion die $\mathrm{FEV}_{1} / \mathrm{VC} \leq 80 \%$ des Sollwertes sein und die $\mathrm{FEV}_{1}$ nach Inhalation eines Beta-2-Sympathomimetikums um weniger als $12 \%$ gegenüber dem Ausgangswert ansteigen. Ausschlusskriterien waren eine respiratorische Insuffizienz, ferner ein Alter von mehr als 75 Jahren.

Zum Zeitpunkt der Schulung sowie ein und zwei Jahre danach wurden die Patienten körperlich untersucht und eine Lungenfunktionsanalyse $\left(\mathrm{FEV}_{1}, \mathrm{FEV}_{1} / \mathrm{VC}, \mathrm{R}_{\mathrm{AW}}\right)$ durchgeführt, außerdem mussten die Patienten einen Fragebogen ausfüllen. Die wesentlichen Inhalte des Fragebogens waren: Anzahl der Phasen mit verstärkter Atemnot; Anzahl der Exazerbationen sowie Hausarztund Notfallbehandlungen pro Jahr; Fähigkeit zur Selbstmedikation bei akuter Exazerbation; Selbstkontrolle der Erkrankung mit Symptom-Beobachtung, Peak-Flow-Messung und Führen eines Patienten-Tagebuches. Zudem mussten die Patienten einen Wissenstest (Multiple-Choice-Test: 20 Fragen) beantworten, um die Kenntnisse über die Erkrankung sowie über die Wirkungen und Nebenwirkungen der verwendeten Medikamente zu prüfen. Von den Krankenkassen wurden Daten eingeholt über die Anzahl der Krankenhausaufenthalte und -tage aufgrund der Diagnosen COPD und aller anderen Diagnosen während des Beobachtungszeitraumes. Eine Analyse der Arbeitsunfähigkeitstage wurde 
nicht vorgenommen, da kein Patient zum Zeitpunkt der Schulung berufstätig war.

\section{Inhalte und Struktur des Schulungsprogramms für Patienten mit COPD}

Als wesentliche Inhalte einer Patientenschulung für chronische Erkrankungen wurden folgende Punkte definiert [7,22]: Vermittlung von Krankheits- und Behandlungswissen, Verbesserung der Krankheits- und Behandlungseinsicht, Stärkung der Eigenverantwortlichkeit, Vermittlung sozialer Fähigkeiten zur Krankheitsbewältigung, Verbesserung der Selbstwahrnehmung, Einübung präventiver Handlungen und Verhalten in Krisensituationen.

Angewendet auf das Krankheitsbild der chronisch obstruktiven Bronchitis bedeutet dies, dass der Patient Verantwortung für die Selbstkontrolle seiner Erkrankung übernimmt: Mit Hilfe von Peak-Flow-Messungen, Beobachtung und Protokollierung von Symptomen wie Husten, Atemnot und Auswurf in Tagebüchern lernt er, rechtzeitig eine Verschlechterung der Stabilität seiner Atemwege zu erkennen und darauf mit medikamentösen und nicht-medikamentösen Selbsthilfemaßnahmen zu reagieren.

Das ambulante Fürther Schulungsprogramm für Patienten mit chronisch obstruktiver Bronchitis und Lungenemphysem (AFBE) besteht aus vier Doppelstunden, der Unterricht wurde in Kleingruppen (4-8 Teilnehmer) durchgeführt. Als wesentliche Unterrichtsmedien wurden Tafel und Flipchart bzw. Overheadfolien eingesetzt. Die Schulungen wurden von Ärzten durchgeführt, die langjährige Erfahrung in der Patientenschulung hatten.

Wesentliche Schulungsinhalte sind: Definition, Ursachen und Symptome der COPD, Rauchen als häufigste Ursache der COPD und die Möglichkeiten zur Raucherentwöhnung, Folgeerkrankungen der COPD, Selbstkontrolle der Erkrankung (Peak-FlowMeter, Ampel-System, Patienten-Tagebuch, Symptombeobachtung), korrekte Inhalationstechnik der Medikamente (Dosieraerosole, Pulverinhalatoren, Düsenvernebler), Wirkungen und Nebenwirkungen der wichtigsten Medikamente (Stufenplan der Deutschen Atemwegsliga 1995 [34]: Beta-2-Sympathomimetika, Anticholinergika, Theophyllin, Kortison inhalativ und systemisch), Sekretelimination (Medikamente, physikalische Maßnahmen), selbständige Dosisanpassung der Medikamente an den Schweregrad der Atemwegsobstruktion, Bronchialinfekt, Verhalten bei akuter Atemnot.

Alle Patienten erhielten ein Peak-Flow-Meter und das PatientenTagebuch der Deutschen Atemwegsliga, außerdem Schulungsunterlagen, in denen die wichtigsten Inhalte der Schulung zusammengefasst sind, zudem schriftliche, individuell angepasste Anweisungen zur Dosisanpassung an den aktuellen Schweregrad der bronchialen Obstruktion und einen schriftlichen Notfallplan. Nach der Schulung wurden die Hausärzte der Patienten über die Schulung und deren Inhalte im Rahmen eines Arztbriefes informiert.

\section{Statistik}

Bei normalverteilten ordinalskalierten Merkmalen kam der t-Test zur Anwendung, bei nicht normalverteilten ordinalskalierten Merkmalen der U-Test nach Wilcoxon, Mann und Whitney sowie bei dichotomen Merkmalen der McNemar VorzeichenTest. Die Nullhypothese („Es besteht kein statistischer Zusammenhang“) wurde verworfen, wenn das ermittelte Signifikanzniveau unter dem Testniveau $(p<0,05)$ lag.

\section{Ergebnisse}

\section{Wissen der Patienten bezüglich ihrer Erkrankung sowie über Wirkungen und Nebenwirkungen der verwendeten Medikamente}

Die Vermittlung von Wissen sowohl über die Erkrankung als auch über die Therapie war ein wesentlicher Bestandteil des Schulungsprogrammes. Zur Überprüfung des Wissenszuwachses wurde ein Multiple-Choice-Fragebogen mit 20 Fragen eingesetzt. Nach der Teilnahme an der Schulung stieg das Wissen der Patienten über die Erkrankung sowie über Wirkungen und Nebenwirkungen der Medikamente signifikant an: Vor der Schulung waren in einem Multiple-Choice-Test 9,1 $\pm 2,1 \%$ (alle Angaben als Mittelwert \pm Standardfehler) der Antworten korrekt, ein Jahr nach der Schulung $68,7 \pm 5,4 \%(p<0,01)$ und nach zwei Jahren $70,5 \pm 4,8 \%(\mathrm{p}<0,01)$.

\section{Lungenfunktion}

Keine signifikante Änderung ergab sich für die Kenngrößen der Atemwegsobstruktion in der Lungenfunktion. Vor der Teilnahme an der Schulung lag der $\mathrm{FEV}_{1}$-Wert bei $1,6 \pm 0,1$, ein Jahr nach der Schulung bei 1,7 $\pm 0,21$ (n.s.) sowie zwei Jahre nach der Schulung bei 1,6 $\pm 0,21$ (n.s.). In gleicher Weise zeigte sich keine signifikante Veränderung des Gesamtatemwegswiderstandes $R_{\mathrm{AW}}$.

\section{Zusammensetzung und Einschätzung der Medikation, korrekte Anwendung der Medikamente, Rauchverhalten} Nach der Teilnahme an der Schulung ergab sich keine Veränderung in der Zusammenstellung der medikamentösen Dauertherapie: Die Substanzgruppen Beta-2-Sympathomimetika, Anticholinergika und Theophyllin wurden mit der gleichen Häufigkeit eingesetzt, ebenfalls keine Änderungen ergaben sich für die inhalativen und systemischen Steroide (Tab. 2).

Tab. 2 Veränderung der Medikation vor, ein und zwei Jahre nach der Schulung (AFBE): Anteil der Patienten in \%

\begin{tabular}{|c|c|c|c|c|c|}
\hline Medikamente & $\begin{array}{l}1 \mathrm{Jahr} \\
\text { vor AFBE }\end{array}$ & $\begin{array}{l}1 \text { Jahr } \\
\text { nach AFBE }\end{array}$ & p-Wert & $\begin{array}{l}2 \text { Jahre } \\
\text { nach AFBE }\end{array}$ & p-Wert \\
\hline $\begin{array}{l}\text { inhalatives kurzwirk- } \\
\text { sames Beta-2-Sym- } \\
\text { pathomimetikum }\end{array}$ & 33,4 & 42,8 & n.s. & 42,1 & n.s. \\
\hline Anticholinergika & 14,3 & 9,5 & n.s. & 15,8 & n.s. \\
\hline $\begin{array}{l}\text { Kombination kurzwirk- } \\
\text { sames Beta-2-Sym- } \\
\text { pathomimetikum mit } \\
\text { Anticholinergikum }\end{array}$ & 61,9 & 57,1 & n.s. & 52,6 & n.s. \\
\hline $\begin{array}{l}\text { orales retardiertes } \\
\text { Beta-2-Sympatho- } \\
\text { mimetikum }\end{array}$ & 28,5 & 42,9 & n.s. & 26,3 & n.s. \\
\hline $\begin{array}{l}\text { retardiertes } \\
\text { Theophyllin }\end{array}$ & 61,9 & 76,2 & n.s. & 68,4 & n.s. \\
\hline inhalatives Steroid & 38,1 & 33,3 & n.s. & 31,6 & n.s. \\
\hline
\end{tabular}


Tab. 3 Verhalten der Patienten während akuter Atemnot vor, ein und zwei Jahre nach der Schulung (AFBE): Anteil der Patienten in \%

\begin{tabular}{|c|c|c|c|c|c|}
\hline Maßnahmen & 1 Jahr vor $A F B E$ & 1 Jahr nach AFBE & $p$-Wert & 2 Jahre nach AFBE & p-Wert \\
\hline Peak-Flow-Messung während akuter Atemnot & 9,5 & 66,7 & 0,01 & 63,1 & 0,01 \\
\hline \multicolumn{6}{|l|}{ Selbstmedikation während akuter Atemnot } \\
\hline $\begin{array}{l}\text { - Inhalation kurzwirksames Beta-2-Sympathomimetika m./o. } \\
\text { Anticholinergikum }\end{array}$ & 61,9 & 71,4 & n.s. & 84,2 & n.s. \\
\hline - Einnahme oraler retardierter Theophyllin-Präparate & 38,1 & 0 & 0,01 & 0 & 0,01 \\
\hline
\end{tabular}

Zudem wurden die Patienten nach ihrer subjektiven Einschätzung der verschiedenen Medikamente befragt. Hierzu mussten die Patienten die Wirkstoffgruppen in ihren Wirkungen und Nebenwirkungen subjektiv auf einer visuellen Analogskala beurteilen (Gesamtlänge 100 mm: unbedenklich 0 Punkte; sehr bedenklich 100 Punkte): Während sich für die inhalativen und retardierten oralen Beta-2-Sympathomimetika-Präparate sowie für retardierte Theophyllin-Tabletten und Kortison-Tabletten keine signifikanten Unterschiede ergaben, verbesserte sich die Einschätzung der inhalativen Steroide signifikant: Vor der Schulung lag der Punkt-Wert bei 43,5 $\pm 5,5$, ein Jahr nach der Schulung bei $20,3 \pm 4,5(p<0,05)$ und zwei Jahre nach der Schulung bei 20,0 \pm $5,7(\mathrm{p}<0,05)$.

Die Verbesserung der Inhalationstechnik war ein weiterer Schwerpunkt der Schulung. Nach der Teilnahme an der Schulung verwendeten die Patienten, die mit kortisonhaltigen Dosieraerosolen therapiert wurden $(n=8)$, deutlich häufiger eine Inhalationshilfe (Spacer): Vor der Schulung benutzten 19,0\% der Patienten eine Inhalationshilfe, ein Jahr nach der Schulung 85,7\% $(\mathrm{p}<0,01)$. Dieser Effekt nahm allerdings zwei Jahre nach der Schulung wieder ab, denn nach zwei Jahren lag der Anteil nur noch bei $38,1 \%$ (n.s.).

Keine signifikante Veränderung ergab sich beim Rauchverhalten. Im ersten Jahr nach der Schulung stellte lediglich ein Teilnehmer von 13 aktiven Rauchern den Nikotinkonsum ein, im zweiten Jahr wiederum ein Teilnehmer.

\section{Verhalten bei akuter schwerer Atemnot}

Die Verhaltensänderung bezüglich der Selbstmedikation bei akuter Atemnot war ein Schwerpunkt der Schulung: Das korrekte Management bei akuter Atemnot wurde in interaktiven Rollenspielen erarbeitet, zum anderen bekam jeder Patient einen schriftlichen Notfallplan ausgehändigt. Die Verhaltensänderung nach der Schulung lässt sich wie folgt zusammenfassen (Tab. 3): Vor der Schulung verwendeten 38,1\% der Patienten orale retardierte Theophyllinpräparate im Akutfall, ein und zwei Jahre nach der Schulung kein Patient mehr. Während Beta-2-Sympathomimetika vor und nach der Schulung gleich häufig eingesetzt wurden, änderte sich allerdings die Anzahl der Hübe: Vor der Schulung inhalierten die Patienten $11 \pm 0,9$ Hübe pro Ereignis, ein Jahr nach der Schulung $6,4 \pm 0,6$ Hübe $(p<0,05)$ und zwei Jahre danach $5,7 \pm 0,5$ Hübe $(p<0,05)$.

\section{Selbstkontrolle der Erkrankung}

Ein wichtiger Schulungsinhalt ist die Verbesserung der Selbstkontrolle der Erkrankung durch die Patienten, um frühzeitig eine Exazerbation zu erkennen und entsprechend reagieren $\mathrm{zu}$ können. Die Häufigkeit einer regelmäßigen Tagebuchführung mit Eintragung der Peak-Flow-Werte und Symptome nahm durch die Schulung zu: Vor der Schulung führten nur 14,3\% der Patienten regelmäßig ein Tagebuch, ein Jahr nach der Schulung 42,9\% ( $p<0,03$ ) und zwei Jahre danach 31,6\% (n.s.). Analog dazu stieg die Anzahl der Patienten, die regelmäßig (mindestens zweimal pro Tag) Peak-Flow-Messungen durchführten: 23,8\% der Patienten vor der Schulung, 51,8\% nach einem Jahr $(\mathrm{p}<0,01)$ und 42,1\% nach zwei Jahren (n.s.). Ebenso wurde häufiger der Peak-Flow nach der Inhalation eines kurzwirksamen Beta2-Sympathikometikums gemessen (vor der Schulung: 14,3\%; nach 1 Jahr: 66,7\%, p <0,01; nach 2 Jahren: 47,4\%, n.s.).

\section{Auswirkungen auf die Morbidität}

Die Häufigkeit von leichten Atemnotattacken, die die Inhalation eines Beta-2-Sympathomimetikums (ggf. in Kombination mit einem Anticholinergikum) erforderlich machten, konnte durch die Patientenschulung deutlich reduziert werden: Die Häufigkeit pro Tag lag vor der Schulung bei 1,6 $\pm 0,5$ Episoden pro Patient, ein Jahr nach der Schulung bei 1,0 $\pm 0,5(p<0,05)$ und zwei Jahre nach der Schulung bei $1,5 \pm 0,5$ (n.s.).

Für eine Exazerbation musste mindestens eines der folgenden Kriterien erfüllt sein: Zunahme der Atemnot, Zunahme der Auswurfmenge oder Veränderung der Konsistenz und Farbe. Für die Gesamtzahl der Exazerbationen ergab sich keine Änderung, allerdings konnten die schweren Exazerbationen, die ärztliche Hilfe erforderten, reduziert werden zugunsten der Exazerbationen, die von den Patienten selbständig bewältigt wurden (Abb.1). In diesem Zusammenhang nahm die Anzahl der von den Patienten initiierten Kortison-Zyklen (Kortison-Stoßtherapie über 3 Wochen in absteigender Dosierung) bei einer akuten Exazerbation deutlich zu: Vor der Schulung führten die Patienten 0,3 $\pm 0,1$ Zyklen pro Jahr durch, im ersten Jahr nach der Schulung 1,0 $\pm 0,3$ $(\mathrm{p}<0,03)$ und im zweiten Jahr 0,7 $\pm 0,2$ (n.s.). Begleitet wurden die häufigeren Kortison-Zyklen durch eine höhere Anfangsdosierung (während der Schulung empfohlene Dosis: 40 mg Prednisolonäquivalente): Vor der Schulung lag die initiale Dosis bei $16,0 \pm 0,1 \mathrm{mg}$ Prednisolon-Äquivalenten, im Jahr danach bei $35,6 \pm 1,8 \mathrm{mg}$ (n.s.) und im zweiten Jahr bei $39,6 \pm 2,5 \mathrm{mg}$ (n.s.) (Tab. 4). 


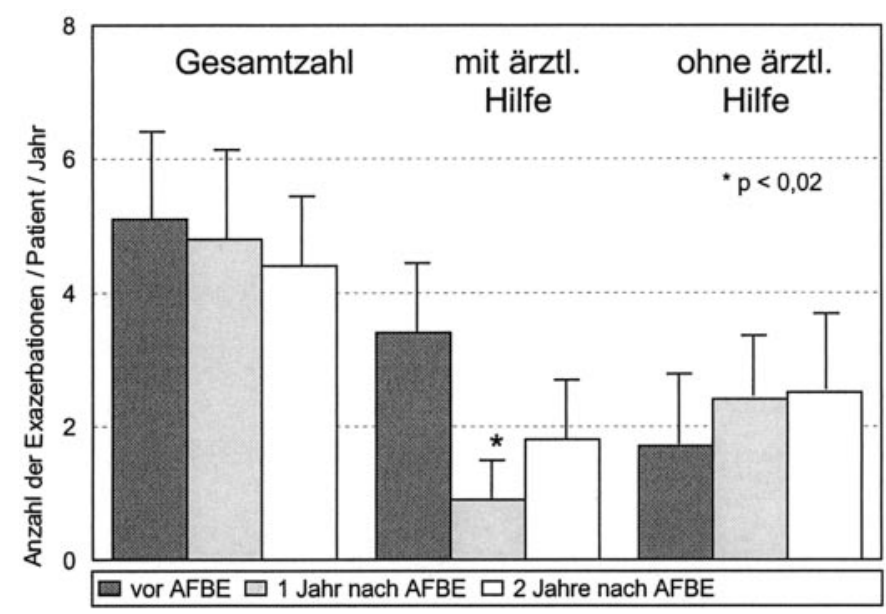

Abb. 1 Anzahl der Exazerbationen pro Patient und Jahr: Gesamtzahl aller Exazerbationen, Anzahl der mit ärztlicher Hilfe bzw. ohne ärztliche Hilfe bewältigten Exazerbationen vor sowie ein und zwei Jahre nach der Schulung (Mittelwert \pm Standardfehler).

Tab. 4 Bronchiale Infekte: Häufigkeit pro Patient und Jahr, Häufigkeit der Peak-Flow-Kontrollen sowie Medikation während des Infektes

\begin{tabular}{|c|c|c|c|c|c|}
\hline & $\begin{array}{l}1 \mathrm{Jahr} \\
\text { vor AFBE }\end{array}$ & $\begin{array}{l}1 \text { Jahr } \\
\text { nach AFBE }\end{array}$ & p-Wert & $\begin{array}{l}2 \text { Jahre } \\
\text { nach AFBE }\end{array}$ & p-Wert \\
\hline $\begin{array}{l}\text { Anzahl der bronchialen } \\
\text { Infekte (Anzahl pro Jahr, } \\
\text { Mittelwert } \pm \text { SEM) }\end{array}$ & $4,5 \pm 1,6$ & $1,8 \pm 0,4$ & 0,05 & $1,4 \pm 0,2$ & 0,01 \\
\hline $\begin{array}{l}\text { Peak-Flow-Messung bei } \\
\text { Infekt (Patienten in \%) }\end{array}$ & 23,8 & 85,7 & 0,01 & 63,3 & n.s. \\
\hline $\begin{array}{l}\text { Häufigkeit der Ein- } \\
\text { nahme systemischer } \\
\text { Steroide } \\
\text { (Patienten in \%) }\end{array}$ & 42,9 & 42,9 & n.s. & 57,9 & n.s. \\
\hline $\begin{array}{l}\text { Höhe der Kortison- } \\
\text { anfangsdosis in (mg) } \\
\text { (Mittelwert } \pm \text { SEM) }\end{array}$ & $13,9 \pm 2,1$ & $33,0 \pm 6,0$ & 0,02 & $39,0 \pm 3,5$ & 0,02 \\
\hline
\end{tabular}

Nach der Patientenschulung nahm die Anzahl der Bronchialinfekte pro Patient und Jahr signifikant ab (Tab. 4). Ein Bronchialinfekt wurde wie folgt definiert: Zunahme der Menge des Auswurfs, Änderung der Konsistenz und Farbe, zudem mussten zusätzliche Infektzeichen wie Fieber und Abgeschlagenheit vorliegen. Vor und nach der Schulung wurden die Bronchialinfekte gleich häufig mit systemischen Steroiden behandelt, wobei allerdings nach der Schulung eine signifikant höhere Anfangsdosis zu verzeichnen war.

\section{Krankenhaustage und -aufenthalte, Arztbesuche}

Die Anzahl der Krankenhaustage und -aufenthalte aufgrund der Diagnose „COPD“ konnte deutlich reduziert werden, hingegen blieb die Anzahl der Krankenhaustage und -aufenthalte aufgrund anderer Diagnosen konstant (Abb. 2).

Die Anzahl der Konsultationen des Hausarztes aufgrund der Diagnose „chronisch obstruktive Bronchitis“ nahm nach der Schulung signifikant ab: Im Jahr vor der Schulung betrug die Anzahl

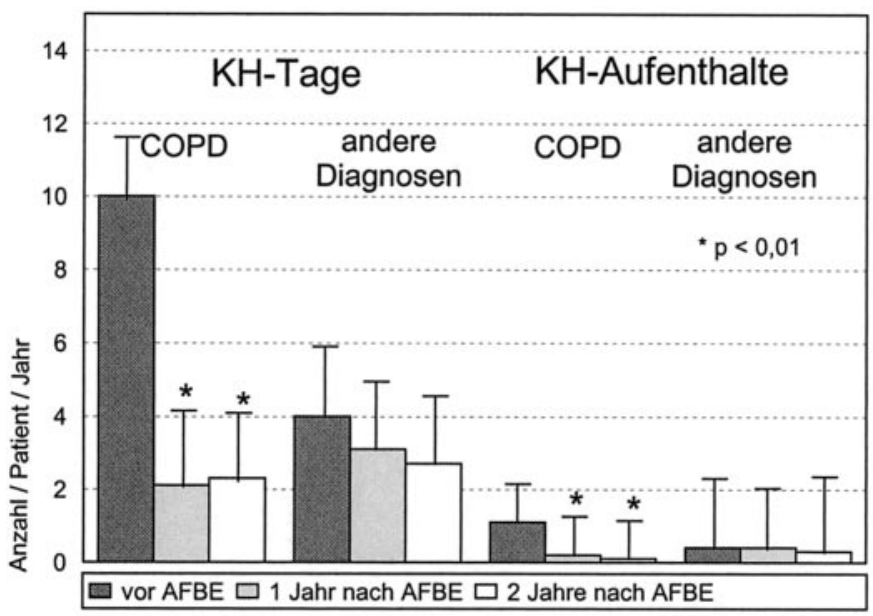

Abb. 2 Anzahl der Krankenhaustage und Krankenhausaufenthalte (KH-Tage bzw. KH-Aufenthalte) pro Patient und Jahr aufgrund der Diagnose COPD und allen anderen Diagnosen, jeweils vor sowie ein und zwei Jahre nach der Schulung (Mittelwert \pm Standardfehler).

der Konsultationen 2,5 $\pm 0,4$ pro Patient und Monat, ein Jahr nach der Schulung $1,3 \pm 0,1(\mathrm{p}<0,01)$ und zwei Jahre danach $1,3 \pm 0,2(P<0,05)$.

\section{Diskussion}

Die Rehabilitation bei chronischen obstruktiven Atemwegserkrankungen wird definiert als „multidimensional continuum of services" [9]. Wesentliche Bestandteile sind neben einer optimierten Pharmakotherapie besonders körperliches Training, Patientenschulung, Atemtherapie sowie psychosoziale Unterstützung $[1,3,5,8,23]$.

Der Nutzen des körperlichen Trainings für COPD-Patienten ist mittlerweile bezüglich einer Steigerung der körperlichen Leistungsfähigkeit und der Lebensqualität gut belegt $[1,3,5,8,14,25]$. Im Gegensatz zum Asthma bronchiale ist bei COPD-Patienten der Stellenwert der Patientenschulung in der Rehabilitation noch nicht hinreichend geklärt $[4,11,26,28,37]$. In einer randomisierten Studie von Ries und Toshima [26,31] nahmen 119 Patienten entweder an einem 8-wöchigen Rehabilitationsprogramm mit körperlichem Training oder an einer Patientenschulung teil: Im Gegensatz zu der Rehabilitationsgruppe verbesserten sich die geschulten Patienten nicht bezüglich der körperlichen Leistungsfähigkeit und der Symptomatik. Andere Untersuchungen kommen zu ähnlichen negativen Ergebnissen $[2,12,19]$. In einer neuseeländischen Studie [33] mit insgesamt 56 Patienten wurden die Teilnehmer in einem Aktionsplan unterwiesen: Bei einer Symptomverschlechterung sollte selbständig eine KortisonStoßtherapie sowie eine Antibiotika-Behandlung eingeleitet werden. Nach 6 Monaten ergaben sich keine Unterschiede zwischen der Kontroll- und der Interventionsgruppe bezüglich der Parameter Lebensqualität, Lungenfunktion, Symptomatik sowie Anzahl der Arztkonsultationen. Allerdings verbesserten sich deutlich die Selbstmanagement-Fähigkeiten: 34\% Patienten in der Interventionsgruppe im Vergleich zu 7\% in der Kontrollgruppe leiteten selbständig bei Symptomverschlechterung eine Korti- 
son-Therapie ein, 44\% im Gegensatz zu 7\% eine Antibiotika-Behandlung.

Die Unterschiede zu den doch ermutigenden Ergebnissen unserer Pilotstudie bedürfen einer näheren Betrachtung: Erstens wurde lediglich eine Pilotstudie mit methodischen Defiziten wie einer kleinen Fallzahl und einer fehlenden Randomisierung in Kontroll- und Interventionsgruppe durchgeführt. Zudem wurden die Parameter Lebensqualität und körperliche Leistungsfähigkeit nicht untersucht. Ferner sind in unserer Schulung im Gegensatz zu anderen Untersuchungen ausschließlich Patienten mit einer leicht- bis mittelgradigen Ausprägung der Erkrankung eingeschlossen worden.

Außerdem wird aus der Literaturübersicht deutlich, dass der Begriff „Schulung“ ein Sammelbegriff für verschiedene Maßnahmen ist: Das Spektrum reicht hier von der eigentlichen Patientenschulung bis hin zu Informationsangeboten (Videos, Broschüren, etc.). Als „Patientenschulung“ im engeren Sinne wird aber ein strukturiertes Schulungsprogramm mit psychomotorischen, affektiven und kognitiven Lernbereichen definiert [7,23]: Vermittlung von Krankheits- und Behandlungswissen, Verbesserung der Krankheits- und Behandlungseinsicht, Stärkung der Eigenverantwortlichkeit, Verbesserung der Selbstwahrnehmung, Einübung präventiver Handlungen und Verhalten in Krisensituationen. Das beinhaltet auch schriftliche, individuell angepasste Handlungsanweisungen zur Dosisanpassung an den aktuellen Schweregrad der bronchialen Obstruktion sowie einen schriftlichen Notfall-Plan [23]. Das Ziel des Patiententrainings ist eine Verhaltensänderung, d.h. die Stärkung der SelbstmanagementFähigkeiten des Patienten [37]. Neben einem strukturierten Schulungsprogramm ist auch die Größe der Schulungsgruppe von entscheidender Bedeutung. Im Vergleich zur Individualschulung ist die Gruppenschulung effektiver, wenn die Kleingruppe aus 4-8 Teilnehmern besteht und die Möglichkeit zur Interaktion und zum Erfahrungsaustausch bietet [35].

Das Ausmaß der Effekte der COPD-Schulung ist nicht vergleichbar mit den Erfolgen von ambulanten und stationären Schulungsprogrammen für erwachsene Asthmatiker [16-18]. Das liegt einmal an der Charakteristik der Erkrankung, die im Gegensatz zum Asthma bronchiale durch eine meist irreversible Atemwegsobstruktion gekennzeichnet ist, außerdem am höheren $\mathrm{Al}$ ter der COPD-Patienten.

Kritisch sind ebenfalls die unzureichenden Erfolge der Raucherentwöhnung [36]. In der Schulung wird auf die verschiedenen Möglichkeiten der Raucherentwöhnung hingewiesen, um die Patienten zur Abstinenz zu motivieren, zudem wurde die Teilnahme an einem gesonderten Programm zur Raucherentwöhnung angeboten. In unserer Pilotstudie wie auch in anderen Untersuchungen waren allerdings die positiven Effekte unabhängig von Änderungen des Nikotinkonsums [2,8], denn das Rauchverhalten hat sich während der zweijährigen Beobachtungszeit nur unwesentlich geändert. Auf ein Raucherentwöhnungsprogramm als fester Bestandteil der Schulung wurde verzichtet, da unter den Schulungsteilnehmern nicht mehr alle aktive Raucher waren, zudem hätte der verhaltenstherapeutische Ansatz der Raucherentwöhnung die Integration eines Psychologen erforderlich gemacht.
Problematisch ist außerdem, dass das Peak-Flow-Meter nicht in gleichem Maße für das Monitoring der COPD wie für das Asthma bronchiale geeignet ist: Mit dem Peak-Expiratory-Flow-Wert (PEF) werden Obstruktionen im Bereich der kleinen Atemwege unterschätzt, und somit eignet sich das Gerät nur für eine Minderheit der COPD-Patienten für das Monitoring der Erkrankung [21]. Um dieses Defizit auszugleichen, wurde in unserer Schulung bei der Selbstkontrolle der Erkrankung mehr Wert auf die Selbstbeobachtung der Symptome wie Husten, Atemnot, Auswurf (Menge, Konsistenz, Farbe) sowie auf die Einschränkung der körperlichen Belastbarkeit gelegt. Neue Möglichkeiten eröffnen hier tragbare elektronische Spirometer, die zusätzlich zum PEF-Wert auch die spirometrischen Messwerte FEV $_{1}$, FVC und $\mathrm{MEF}_{25-75}$ erfassen.

Zusammenfassend bleibt festzuhalten, dass Patientenschulungen, insbesondere auch ein ambulantes Programm, für Patienten mit leicht- bis mittelgradiger chronisch obstruktiver Bronchitis effektiv sein können. Neben einer Reduktion der Morbidität und einer Verbesserung der Selbstmanagement-Fähigkeiten der Patienten ist auch ein ökonomischer Nutzen durch die Reduktion der Bronchialinfekte sowie der Krankenhaustage und -aufenthalte zu erwarten [29,32]. Die Schulungseffekte sind auch noch zwei Jahre nach der Teilnahme an dem Programm nachweisbar, wobei allerdings bei der Selbstkontrolle der Erkrankung ein deutlicher Abfall zu verzeichnen ist. Aus diesem Grund erscheinen Refresher-Kurse im jährlichen Abstand sinnvoll.

Umfassendere Studien an größeren Patientenkollektiven mit unterschiedlichen Schweregraden der Erkrankung, einem längeren Beobachtungszeitraum und mit Einbeziehung der Aspekte Lebensqualität und Krankheitsbewältigung sind notwendig, um den endgültigen Stellenwert der Patientenschulung im Management der COPD zu bestimmen und um den Einfluss auf die Prognose der Erkrankung zu untersuchen.

\section{Literatur}

${ }^{1}$ Ambrosini N, Vitacca M, Rampulla C. Standards for rehabilitative strategies in respiratory diseases. Monaldi Arch Chest Dis 1995; 50: $293-318$

${ }^{2}$ Ashikaga T, Vacek PM, Lewis SO. Evaluation of a community-based education programme for individuals with chronic obstructive pulmonary disease. J Rehabil Res Dev 1980; 46: 23 - 27

${ }^{3}$ Clark CJ. Pulmonary rehabilitation. In: Calverey P, Pride N (Hrsg.). Chronic obstructive pulmonary disease. London: Chapman and Hall, 1995

${ }^{4}$ Clark CJ. The place of education in the management of chronic obstructive lung disease. Monaldi Arch Chest Dis 1994; 49: 68 - 70

${ }^{5}$ Celli BR. Pulmonary rehabilitation in patients with COPD. Am J Respir Crit Care Med 1995; 152: 861 - 864

${ }^{6}$ Celli BR. Standards for the optimal management of COPD: a summary. Chest 1998; 113: 5283-5287

${ }^{7}$ Creer TL. The application of behavioral procedures to childhood asthma: current and future perspectives. Patient Educ Couns 1992; 17: $9-22$

${ }^{8}$ Donner $\mathrm{CF}$, Howard P. Pulmonary rehabilitation in chronic obstructive pulmonary disease (COPD) with recommendations for its use. Eur Respir J 1992; 5: 266-275

${ }^{9}$ Fishman AP, NIH workshop summary. Pulmonary rehabilitation research. Am J Respir Crit Care Med 1994; 149: 825-833

${ }^{10}$ Folgering H, Rooyakkers J, von Herwaarden C. Education and cost/benefit ratios in pumonary patients. Monaldi Arch Chest Dis 1994; 49: $166-168$ 
${ }^{11}$ Goldstein R, Lacasse Y, Guyatt G. Controlled studies of pulmonary rehabilitation. ATS/ALA postgraduale workshop summary: Pulmonary rehabilitation, update 1997. San Francisco, 1997

${ }^{12}$ Howland J, Nelson EG, Barlow PB et al. Chronic obstructive airway disease. Impact of health education. Chest 1986; 90: 233-238

${ }^{13}$ Konietzko N, Fabel H. Weißbuch Lunge 2000. Stuttgart, New York: Thieme

${ }^{14}$ Lacasse Y, Wong E, Gyuatt GH et al. Meta-analysis of respiratory rehabilitation in chronic obstructive pulmonary disease. Lancet 1996; 348: $1115-1119$

${ }^{15}$ Mayo PH, Weinberg DJ, Kramer B et al. Results of a programme to improve the process of inpatient care of adult asthmatics. Chest 1996; 110: $48-52$

${ }^{16}$ Mühlhauser l. Die Bedeutung der Patientenschulung in der Behandlung chronischer Krankheiten: Diabetes mellitus, Hypertonie, Asthma bronchiale. Internist Welt 1987; 8: 1-13

${ }^{17}$ Mühlhauser l, Richter B, Kraut D et al. Evaluation of a structured treatment and teaching programme on asthma. J Int Med 1991; 230: $157-164$

${ }^{18}$ Münks-Lederer C, Dhein Y, Richter B et al. Evaluation eines strukturierten ambulanten Asthma-Behandlungs- und Schulungsprogrammes (AFAS). Eine Pilotstudie. Pneumologie 2001; 55: 84-90

${ }^{19}$ Neish CM, Hopp JW. The role of education in pulmonary rehabilitation. J Cardiopulm Rehabil 1988; 11: 439-441

${ }^{20}$ Nolte D. Epidemiologie der chronisch obstruktiven Bronchitis. In: Konietzko N (Hrsg.). Bronchitis. München: Urban \& Schwarzenberg, 1995

${ }^{21}$ Pedersen OF. The Peak Flow Working Group: physiological determinants of peak. Eur Respir J Suppl 1997; 24: S11 - 16

22 Petro W. Patiententraining bei obstruktiven Atemwegserkrankungen - die Situation in Deutschland. Pneumologie 1993; 47: 26-29

${ }^{23}$ Petro W, Wettengel R, Worth $\mathrm{H}$ et al. Empfehlungen zum strukturierten Patiententraining bei obstruktiven Atemwegserkrankungen. Pneumologie 1995; 49: 455-460

${ }^{24}$ Rampulla C, Bruschi C. International comparison in COPD morbidity and mortality. Monaldi Arch Chest Dis 1993; 48: 389-394
${ }^{25}$ Ries AL. Rehabilitation for the patient with advanced lung disease: Designing appropiate programs, establishing realistic goals, meeting the goals. Sem Respir Crit Care Med 1996; 17: 451 - 463

${ }^{26}$ Ries AL, Kaplan RM, Linberg TM et al. Clinical trial of pulmonary rehabilitation versus education in COPD. Am Rev Respir Dis 1994; 149: 595 -

27 Siafakas NM, Vermeire P, Pride NB et al. ERS-Consensus statement, optimal assessment und management of chronic obstructive pulmonary disease (COPD). Eur Respir J 1995; 8: 1398 - 1420

${ }^{28}$ Spohn S, Wittmann M, Petro W. Patientenverhaltenstraining bei COPD: Konzepte und Effizienz. Pneumologie 2000; 54: 72 - 79

${ }^{29}$ Toevs CD, Kaplan RM, Atkins CJ. The costs and effects of behavioral programs in chronic obstructive pulmonary disease. Med Care 1984; 22: $1088-1100$

${ }^{30}$ Trautner C, Richter B, Berger M. Cost-effectiveness of a structured treatment and teaching programme on asthma. Eur Respir J 1993; 6: $1485-1491$

${ }^{31}$ Toshima MT, Kaplan RM, Ries AL. Experimental evaluation of rehabilitation in chronic obstructive pulmonary disease: short-term effects on exercise endurance and health status. Health-Psychol 1990; 9: $237-252$

32 Tuogaard L, Krone T, Sorknaes A et al. Economic benefits of teaching patients with chronic obstructive pulmonary disease about their illness. Lancet 1992; 339: 1517 - 1520

${ }^{33}$ Watson PB, Town GI, Holbrook $\mathrm{N}$ et al. Evaluation of a self-management plan for chronic obstructive pulmonary disease. Eur Respir J 1997; 10: $1267-1271$

${ }^{34}$ Wettengel R, Böhning W, Cegla U et al. Empfehlungen der Deutschen Atemwegsliga zur Behandlung von Patienten mit chronisch obstruktiver Bronchitis und Lungenemphysem. Med Klin 1995; 90: 3-7

${ }^{35}$ Wilson SR. Individual versus group education: Is one better? Pat Educ Couns 1997; 32: $367-375$

${ }^{36}$ Worth H. Smoking cessation. In: Simonds AK, Muir JF, Pierson DJ (Hrsg.). Pulmonary Rehabilitation. London: BMJ Publishing Group, 1996

${ }^{37}$ Worth H. Self management in COPD: one step beyond? Pat Educ Couns 1997; 32: 105-109 